\title{
Synthesis, Morphology, and Optical Properties of Au/CdS Hybrid Nanocomposites Stabilized by Branched Polymer Matrices
}

\author{
V. A. Chumachenko, ${ }^{1}$ A. P. Naumenko, ${ }^{2}$ O. A. Yeshchenko, ${ }^{2}$ \\ N. V. Kutsevol, ${ }^{1}$ and I. S. Bondarchuk ${ }^{2}$ \\ ${ }^{1}$ Faculty of Chemistry, Taras Shevchenko National University of Kyiv, 60 Volodymyrs'ka Street, Kyiv 01601, Ukraine \\ ${ }^{2}$ Faculty of Physics, Taras Shevchenko National University of Kyiv, 60 Volodymyrs'ka Street, Kyiv 01601, Ukraine
}

Correspondence should be addressed to V. A. Chumachenko; chumachenko_va@ukr.net

Received 28 January 2016; Revised 22 March 2016; Accepted 4 April 2016

Academic Editor: William Yu

Copyright (C) 2016 V. A. Chumachenko et al. This is an open access article distributed under the Creative Commons Attribution License, which permits unrestricted use, distribution, and reproduction in any medium, provided the original work is properly cited.

\begin{abstract}
Metal/semiconductor $(\mathrm{Au} / \mathrm{CdS})$ nanocomposites were synthesized in the solution of branched D-g-PAA polymer. TEM and DLS of $\mathrm{Au} / \mathrm{CdS} / \mathrm{D}$-g-PAA nanocomposites revealed complicated nanocomposite structure consisting of the Au nanoparticles (NPs) of $6 \mathrm{~nm}$ in size surrounded by small CdS NPs with size of $3 \mathrm{~nm}$. These nanocomposites formed the aggregates-clusters with average size of 50-800 nm. Absorption spectra of Au/CdS nanocomposites consist of the bands of excitons in CdS NPs and surface plasmons in $\mathrm{Au}$ ones. The surface plasmon band of gold NPs is red shifted and broadened in Au/CdS/D-g-PAA nanocomposites comparing to the one of $\mathrm{Au}$ NPs in $\mathrm{Au} / \mathrm{D}$-g-PAA proving the fact of close location of CdS and Au NPs in the synthesized Au/CdS/D-g-PAA nanocomposites. The PL spectra of $\mathrm{Au} / \mathrm{CdS}$ nanocomposites originate from the radiative transitions in excitons in CdS NPs. The 4-fold increase of intensity of free exciton PL is observed for CdS NPs in Au/CdS/D-g-PAA comparing to CdS ones in CdS/D-gPAA that is due to PL enhancement by local field of surface plasmons of Au NPs. Also, the 12-fold decrease of intensity of localized exciton PL is observed for CdS NPs in Au/CdS/D-g-PAA comparing to CdS ones in CdS/D-g-PAA. Most probably, it is due to passivation of the surface of CdS NPs carried out by the Au ones.
\end{abstract}

\section{Introduction}

Semiconductor nanoparticles are of great interest for both fundamental research and industrial development because of their exciting utilization in the areas of light-emitting diodes, electroluminescent devices, lasers, hydrogen producing catalyst, biological labeling, and immune diagnosis [1-7]. On the other hand, in recent decades, there has been a huge interest in metal-based plasmonic nanostructures for applications in biosensing, nanophotonics, and enhanced light emission [810]. This is due to the collective coherent excitations of the free electrons in nanosized metal, also known as surface plasmon resonance (SPR). Surface plasmons (SPs) give rise to the enhancement of the local electric field close to the surface of metal NPs. Such strong local electric field enhances the optical processes such as surface enhanced Raman scattering
(SERS) [11-13] and surface enhanced photoluminescence (SEPL) [14-18] in molecules, clusters, and NPs of dielectrics and semiconductors located near the surface of metal NPs or close to a rough metal surface. If the molecule is close to the metal NP, the metal NP couples to the molecule through the plasmonic field. So, the metal NP acts as a nanoantenna in the sense that it channels the excitation light to the molecule and directs its photoluminescence (PL) to the far field more efficiently as compared to the free molecule.

Recently, the growing ability to fabricate metal/semiconductor $(\mathrm{M} / \mathrm{S})$ composites, $\mathrm{Au} / \mathrm{CdS}$ ones in particular, on a nanoscale has opened new opportunities for designing multifunctional materials [19-25]. The use of these new materials then requires identifying and understanding the emergence of new properties due to the combination of different material systems on the nanometer length scale. The optical properties 
of these nanostructures, for example, often exhibit interesting deviations from their individual components. These optical effects include a shift in the surface plasmon resonance of noble metal NPs when combined or coated with other materials $[26,27]$ or changes in the PL intensity of semiconductor NPs which can be attributed to the overlap of the electronic structures of the different components [28]. Multicomponent hybrids show advantages over single component materials due to the external light activity benefiting from the synergistic effect among different components. A popular approach to increasing the visible light activity is to integrate the metal NPs with strong plasmonic feature into the multicomponent nanostructures. One attraction of such approach is that the plasmonic metal NPs could serve as sensitizers, enabling the visible light utilization by wide bandgap semiconductors. The plausible design/fabrication strategies are still somewhat unclear and under intensive debating and investigating.

Current paper presents the characterization of $\mathrm{Au}, \mathrm{CdS}$ NPs, and Au/CdS hybrid nanocomposites synthesized into branched D-g-PAA polymer matrices. The size and shape of NPs are actually controlled by the choice of the synthesis route, the protection agent, and the synthesis conditions [26]. Due to high reactivity of NPs the preparation of stable nanosystems requires a protection agent preventing a possible aggregation process [29]. Polymers are commonly used as stabilizing agents. They enhance the stability of NPs by introducing steric and/or electrostatic repulsion between them. On the other hand, polymers can also play a role in the process of the in situ NP formation, namely, in the control of their size and morphology. In our previous research it was shown that branched polymers are more efficient in this aim in comparison with linear analogues [30-33]. The comparative analysis of the optical properties of Au/D-g-PAA, CdS/D-g-PAA, and $\mathrm{Au} / \mathrm{CdS} / \mathrm{D}$-g-PAA was performed. The mutual influence of $\mathrm{Au}$ and CdS NPs on their absorption and PL spectra was observed. In particular, we observed the sufficient enhancement of the free exciton photoluminescence in CdS NPs by the plasmonic field of Au NPs. Also, the quenching of the luminescence of localized excitons in CdS NPs occurring due to possible passivation of the surface of CdS ones was observed.

\section{Experimental Details}

2.1. Materials. $\mathrm{CdSO}_{4}, \mathrm{Na}_{2} \mathrm{~S}, \mathrm{HAuCl}_{4}$, and $\mathrm{NaBH}_{4}$ were purchased from Aldrich and used without additional purification. Branched star-like copolymer dextran-g-polyacrylamide (D-g-PAA) was used as a matrix for in situ $\mathrm{CdS}$ and $\mathrm{Au} / \mathrm{CdS}$ synthesis. The polymer plays role of matrix to act as nucleating, capping, and stabilizing agent simultaneously.

The copolymer was obtained by free radical polymerization initiated by ceric ion reducing agent. Namely, copolymer polyacrylamide chains were grafted onto dextran core $\left(M_{w}=\right.$ $\left.7 \times 10^{4} \mathrm{~g} / \mathrm{mol}\right)[34,35]$. The redox process initiates free radical sites exclusively on the polysaccharide backbone, thus preventing from the formation of PAA homopolymers.

Synthesis, identification, and analysis of the polymer average structure were described in $[34,35]$. The theoretical numbers of grafting sites per dextran backbone for the samples we use in the present work are equal to 5, so the related D-g-PAA copolymers are referred to as D70-g-PAA5. The molecular characteristics of branched polymer obtained by size exclusion chromatography were the following: $M_{w}=$ $1.57 \times 10^{6} \mathrm{~g} / \mathrm{mol} ; M_{w} / M_{n}=1.81$; and $R_{g}=85 \mathrm{~nm}$.

This copolymer has star-like structure, consisting of a compact dextran core and long polyacrylamide arms [34]. The structure peculiarities of branched polymers, causing higher local concentration of functional groups in comparison with their linear analogue, give the advantages for application of branched polymer systems in nanotechnologies [36].

2.2. Polymer Characterization. Size exclusion chromatography (SEC) was carried out by using a multidetection device consisting of a LC-10AD Shimadzu pump (throughput $0.5 \mathrm{~mL} / \mathrm{min}$ ), an automatic injector WISP 717+ from Waters, 3 coupled $30 \mathrm{~cm}$ Shodex OH-pak columns (803HQ, 804HQ, and $806 \mathrm{HQ}$ ), a multiangle light scattering detector DAWN $\mathrm{F}$ from Wyatt Technology, and a differential refractometer R410 from Waters. Distilled water containing $0.1 \mathrm{M} \mathrm{NaNO}_{3}$ was used as eluent.

2.3. Synthesis of $A u / D-g-P A A$ and CdS/D-g-PAA Nanocomposites. Au NPs were synthesized by the chemical reduction of $\mathrm{Au}$ precursor $\left(\mathrm{HAuCl}_{4}\right)$ dissolved in polymer aqueous solution ( $\mathrm{pH}=5.5$ ). $0.24 \mathrm{~mL}$ of $0.1 \mathrm{M} \mathrm{HAuCl}_{4}$ aqueous solution was added to $10 \mathrm{~mL}$ of aqueous polymer solution $(c=2 \times$ $10^{-3} \mathrm{~g} / \mathrm{cm}^{3}$ ) and stirred for $20 \mathrm{~min}$. Then, $0.94 \mathrm{~mL}$ of aqueous solution of $\mathrm{NaBH}_{4}\left(0.1 \mathrm{M}, 3.8 \times 10^{-3} \mathrm{~g} / \mathrm{cm}^{3}\right)$ was added. The final aqueous solution was stirred for $30 \mathrm{~min}$. It turned rubyred in colour; thus the formation of Au NPs was indicated.

CdS NPs synthesis was carried out in the solution of polymer at room temperature $\left(25^{\circ} \mathrm{C}\right)$. Stock solution of $\mathrm{CdSO}_{4}$ $\left(0.1 \mathrm{M}, 2.084 \times 10^{-3} \mathrm{~g} / \mathrm{cm}^{3}\right)$ and $\mathrm{Na}_{2} \mathrm{~S}\left(0.1 \mathrm{M}, 7.8 \times 10^{-3} \mathrm{~g} / \mathrm{cm}^{3}\right)$ in deionized water was prepared. $0.9 \mathrm{~mL}$ of $\mathrm{CdSO}_{4}$ solution was added to $20 \mathrm{~mL}$ of aqueous polymer solution ( $c=$ $10^{-3} \mathrm{~g} / \mathrm{cm}^{3}$ ), the mixture was stirred for 15 minutes, and then $0.9 \mathrm{~mL}$ of the $\mathrm{Na}_{2} \mathrm{~S}$ stock solution was injected under stirring. The resulting stable yellow CdS colloids were characterized in $24 \mathrm{~h}$ and then remained essentially unchanged for at least 4 months.

2.4. Synthesis of Au/CdS/D-g-PAA Hybrid Nanocomposites. In the first stage the Au NPs were synthesized by the chemical reduction of $\mathrm{Au}$ precursor $\left(\mathrm{HAuCl}_{4}\right)$ dissolved in polymer aqueous solution at $T=25^{\circ} \mathrm{C}$ in aqueous solutions of dextrangraft-polyacrylamide copolymer (D70-g-PAA5). $0.24 \mathrm{~mL}$ of $0.1 \mathrm{M} \mathrm{HAuCl}_{4}$ aqueous solution was added to $10 \mathrm{~mL}$ of aqueous polymer solution $\left(c=2 \times 10^{-3} \mathrm{~g} / \mathrm{cm}^{3}\right)$ and stirred for $20 \mathrm{~min}$. Then, $0.94 \mathrm{~mL}$ of aqueous solution of $\mathrm{NaBH}_{4}(0.1 \mathrm{M}$, $3.8 \times 10^{-3} \mathrm{~g} / \mathrm{cm}^{3}$ ) was added. The final aqueous solution was stirred for $30 \mathrm{~min}$. It turned ruby-red in colour; thus the formation of Au NPs was indicated. Then $0.9 \mathrm{~mL}$ of $\mathrm{CdCl}_{2}$ solution was added to $5 \mathrm{~mL}$ Au precursor solution, the mixture was stirred for 15 minutes, and then $0.9 \mathrm{~mL}$ of the $\mathrm{Na}_{2} \mathrm{~S}$ stock solution was injected under vigorous stirring. The resulting 


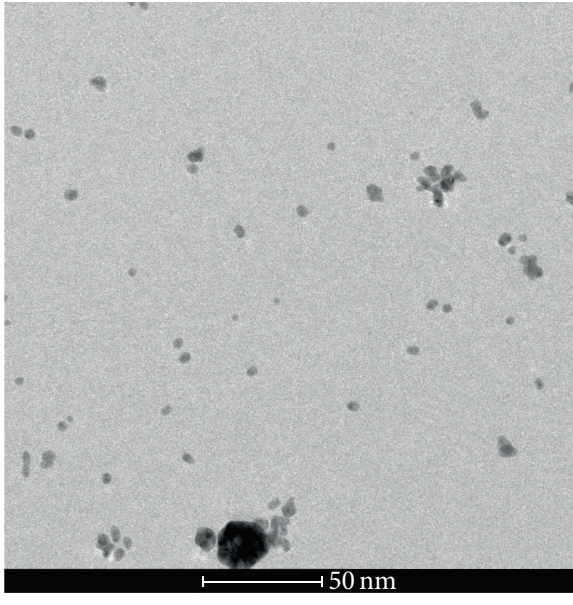

(a)

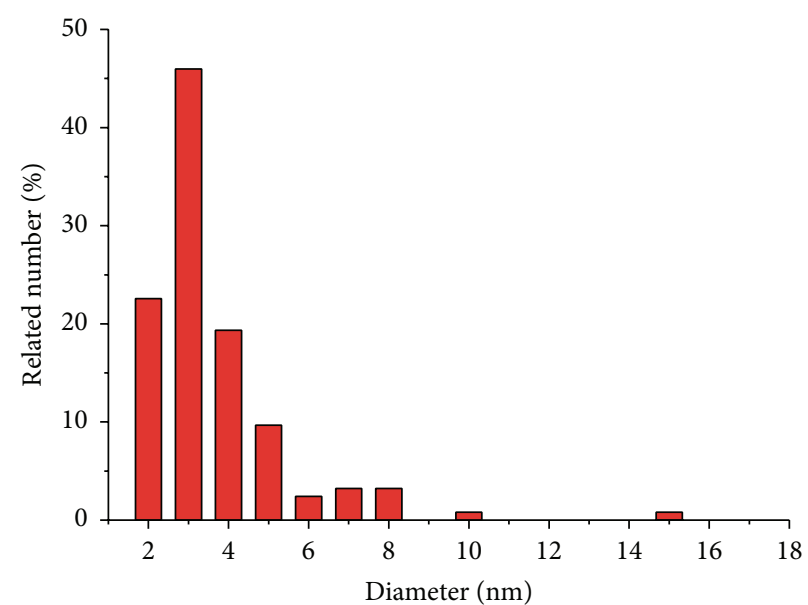

(b)

FIGURE 1: TEM image of Au NPs synthesized in D-g-PAA matrix (a) and the respective size distribution histogram (b).

stable dark-brown colloids were characterized in $24 \mathrm{~h}$ and then remained stable in 4 months.

\subsection{Characterization}

2.5.1. Transmission Electron Microscopy. For the sample preparation 400-mesh $\mathrm{Cu}$ grids with plain carbon film were rendered hydrophilic by a glow discharge treatment (Elmo, Cordouan Technologies, Bordeaux, France). A $5 \mu \mathrm{L}$ drop was deposited and left to adsorb for $1 \mathrm{~min}$; then the excess of solution was removed with a piece of filter paper. The observations of the Au NPs were carried on two TEMs, Tecnai G2 or CM12 (FEI, Eindhoven, Netherlands) and the images were acquired with ssCCD Eagle camera on the Tecnai and a MegaView SIS Camera on the CM12. Image analysis was carried out using the open-source software ImageJ. The NPs' diameters $(d)$ were estimated from NPs' areas $(S)$ as $d=2 \sqrt{S / \pi}$. Over a dozen of TEM images and at least 300 particles for each sample were processed for size distribution analysis in order to obtain reliable data. Gauss fitting was carried out to estimate mean radius and standard deviation of the distribution.

2.5.2. Dynamical Light Scattering (DLS). DLS measurements were carried out using Zetasizer Nano ZS90 (Malvern Instruments Ltd., UK). The apparatus contains a $4 \mathrm{~mW} \mathrm{He}-\mathrm{Ne}$ laser with a wavelength $632.8 \mathrm{~nm}$ and the scattered light is detected at an angle $60^{\circ}$. To estimate hydrodynamic radius distributions from correlation functions, CONTIN algorithm [37], known to be applicable for complicate multicomponent systems [38], was applied using MATLAB code. At least 10 correlation functions were processed for each sample.

2.5.3. Optical Spectroscopy. A tungsten-halogen incandescent lamp was used as the light source for absorption measurements. The single-grating spectrometer MDR-3 was used for the registration of absorption spectra. PL spectra of the samples were measured using Cary Eclipse (Varian Inc.) fluorescence spectrophotometer. The PL was excited by a single monochromatic line with wavelength of $300 \mathrm{~nm}$ (spectral half-width of $5 \mathrm{~nm}$ ) cut from the continuous emission spectrum of a pulse Xe lamp. The repetition rate of the pulses was $80 \mathrm{~Hz}$, pulse duration was $2 \mu$ s, and pulse power density was $1.3 \times 10^{3} \mathrm{~W} / \mathrm{cm}^{2}$. Original solutions were diluted 25 times before spectral measurements.

\section{Results and Discussion}

3.1. Morphology. TEM images of Au and CdS sols as well as $\mathrm{Au} / \mathrm{CdS}$ nanocomposite are represented in Figures $1-3$. The individual sols of $\mathrm{Au}$ and $\mathrm{CdS}$ were prepared and studied to understand the $\mathrm{Au} / \mathrm{CdS}$ nanocomposites structure. The TEM images of Au sol (Figure 1(a)) reveal the existence of the single particles of $3 \pm 0.8 \mathrm{~nm}$ in size and their aggregates with size up to $60 \mathrm{~nm}$, respectively. The TEM images of CdS sols confirm the presence of the single $\mathrm{CdS}$ particles of $5 \pm 1.5$ in size as well as the aggregates of percolated particles (Figures 2(b) and 2(c)). Individual CdS NPs are well defined and are nearly spherical (Figure 1(b)). It is evident that single CdS NPs are not dispersed regularly in the sol but form local structuresclusters with average size of $50-200 \mathrm{~nm}$ (Figure 2(c)). TEM image analysis of $\mathrm{Au} / \mathrm{CdS}$ sols revealed complicated nanocomposite structure (Figure 3(a)). We can observe the $\mathrm{Au}$ NPs of $6 \pm 1.2 \mathrm{~nm}$ in size surrounded by small CdS particles with size of $3 \pm 1 \mathrm{~nm}$ (Figures 3(b) and 3(c)). These nanocomposites form the aggregates-clusters (Figure 1(a)) with average size of 50-800 $\mathrm{nm}$. The Au NPs are well defined (dark grey) in the $\mathrm{Au} / \mathrm{CdS}$ nanocomposite and have spherical shape. The CdS NPs are grey and dispersed around Au NPs.

DLS results evaluated for these nanosystems are shown in Figure 4. DLS results analysis for all nanosystems synthesized in branched polymer matrix (Figure $4(\mathrm{a})$ ) is in good agreement with TEM (Figures 1-3). Synthesized sol included 

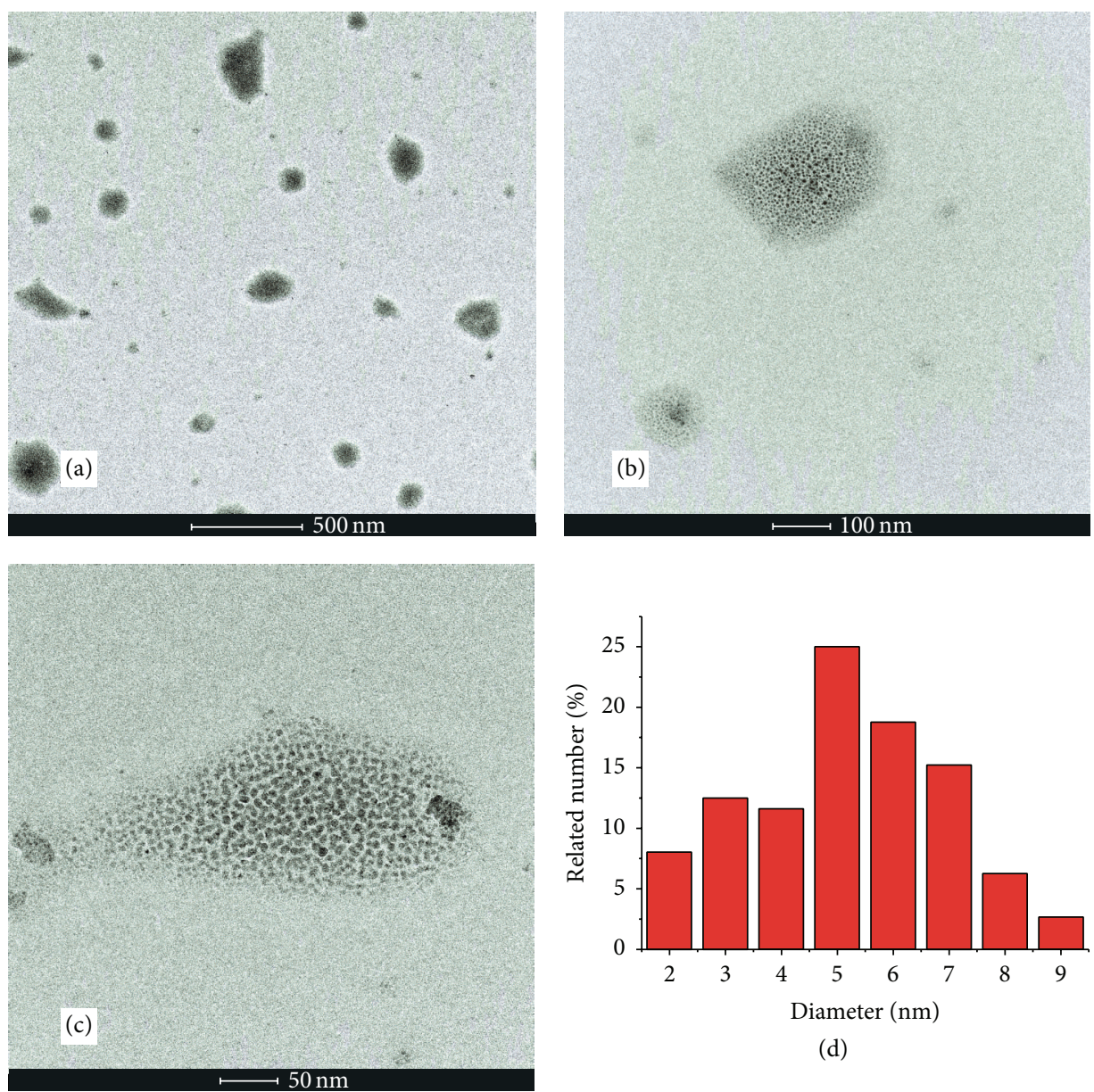

(d)

FIgURE 2: TEM images of CdS NPs synthesized in D-g-PAA matrix (a-c) and the respective size distribution histogram (d).

2 fractions of Au NPs, namely, $3 \pm 1 \mathrm{~nm}$ and $24 \pm 4 \mathrm{~nm}$. For $\mathrm{CdS}$ sols the existence of aggregates having average size of $20-80 \mathrm{~nm}$ (Figure $4(\mathrm{~b})$ ) is observed. That confirmed the TEM results. Taking into account TEM images, DLS results analysis, molecular parameter of polymer $\left(R_{g}=85 \mathrm{~nm}\right)$, and synthesis condition, it can be suggested that the single CdS NPs have been grown directly in the polymer molecule and form the clusters localized in limited macromolecule volume. DLS results for $\mathrm{Au} / \mathrm{CdS}$ nanocomposites reveal the existence in the sols of the aggregates-cluster from 20 to $1000 \mathrm{~nm}$ in size (Figure 4(c)). It can be also concluded that $\mathrm{Au} / \mathrm{CdS}$ nanocomposites are fabricated into the polymer molecule and form the clusters localized in limited macromolecule volume.

3.2. Optical Properties. The light absorption and photoluminescence (PL) spectroscopy was used for optical characterization of fabricated nanocomposites. Room temperature optical absorption spectrum of the CdS NPs in D-g-PAA (Figure 5, blue dotted line) exhibits two bands labeled as $\mathrm{XA}_{\mathrm{s}}$ and $\mathrm{XA}_{\mathrm{a}}$ with maxima at $415 \mathrm{~nm}$ and $457 \mathrm{~nm}$, respectively. These bands are due to the transition in CdS NPs between the ground and first excited exciton states in single NPs and aggregated ones correspondingly [39-41]. Also, it is seen from Figure 5 that the absorption spectrum of Au NPs in D-g-PAA (Figure 5, green dashed line) demonstrates the clear surface plasmon resonance (SPR) band [42-44] with maximum at $536 \mathrm{~nm}$. The absorption spectrum of $\mathrm{Au} / \mathrm{CdS} / \mathrm{D}$-g-PAA hybrids dispersed in water is shown in Figure 5 (red solid line). This spectrum exhibits three absorption bands with maxima at $548 \mathrm{~nm}$, $417 \mathrm{~nm}$, and $460 \mathrm{~nm}$ which are SPR, $\mathrm{XA}_{\mathrm{s}}$, and $\mathrm{XA}_{\mathrm{a}}$ ones corresponding to SPR excitation in Au NPs as well as to excitonic transitions in single and aggregated CdS NPs.

Let us note that the surface plasmon band of Au NPs in $\mathrm{Au} / \mathrm{CdS} / \mathrm{D}$-g-PAA hybrid nanostructures is red shifted (548 nm) comparing to Au NPs in Au/D-g-PAA (536 nm); that is due to the sensitivity of SPR to changes in the dielectric permittivity of the surrounding medium $\varepsilon_{m}$. Indeed, the dielectric permittivity of CdS is higher than the one of polymer. Since the SPR frequency $\omega_{\mathrm{SPR}}$ depends on $\varepsilon_{m}$ as $\omega_{\mathrm{SPR}}=$ $\omega_{p} / \sqrt{1+2 \varepsilon_{m}}$, where $\omega_{p}$ is the bulk plasmon frequency, the increase in $\varepsilon_{m}$ leads to decrease of $\omega_{\mathrm{SPR}}$, that is, to the red shift of SPR. Thus, the absorption spectrum indicates that in $\mathrm{Au} / \mathrm{CdS} / \mathrm{D}$-g-PAA hybrid nanostructures the Au NPs are surrounded by $\mathrm{CdS}$ ones. This conclusion is in agreement with TEM images (Figure 3(a)) showing that small CdS NPs are grouped around larger Au ones. Also, it is seen from Figure 5 that the plasmonic absorption band decreases in intensity and broadens in comparison with sole Au NPs in Au/D-g-PAA 


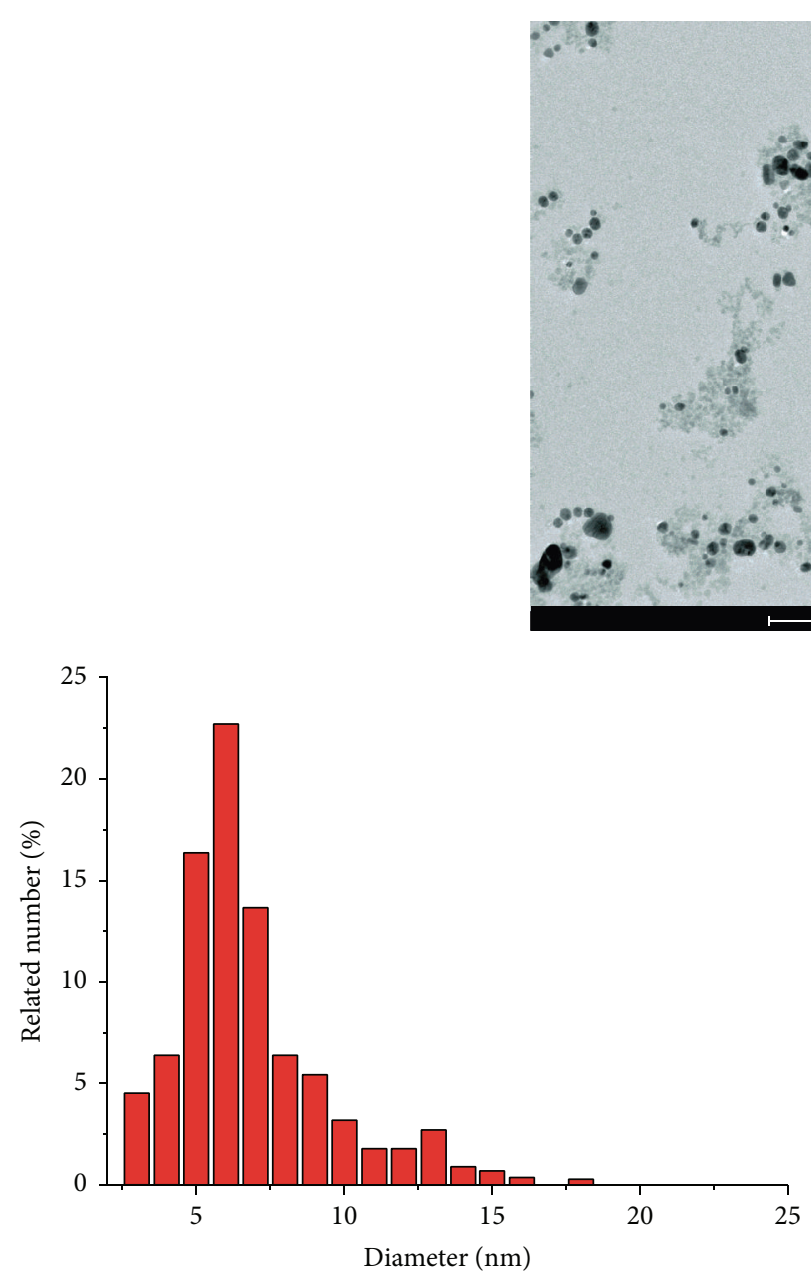

(b)

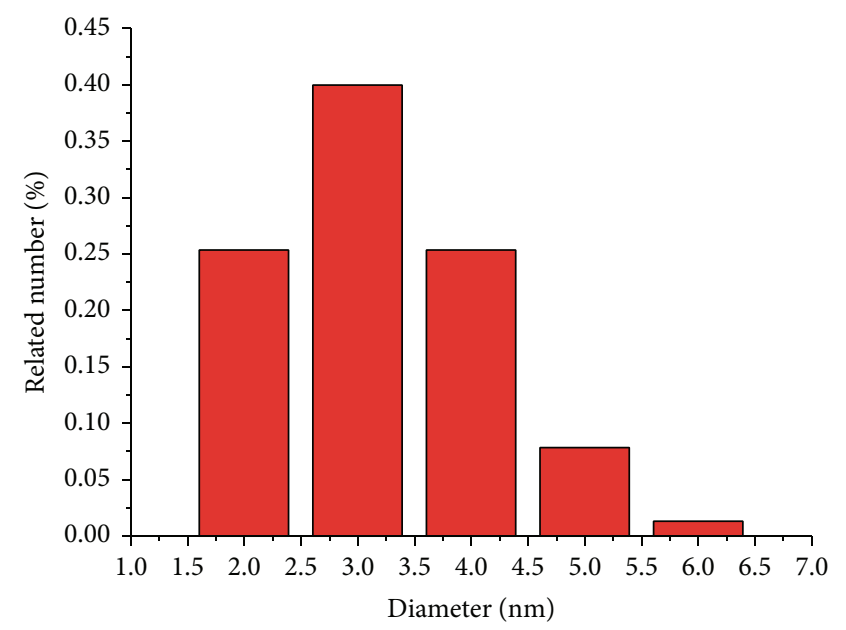

(c)

FIGURE 3: TEM image of Au/CdS sols synthesized in D-g-PAA matrix (a) and the respective size distribution histogram for Au NPs (b) and CdS ones (c).

system. This indicates the fact of increase of plasmon damping. It is known that increase of the plasmon-exciton coupling strength can result in the enhancement of the absorption in CdS NPs and the suppression of the plasmon absorption in $\mathrm{Au}$ NPs [45]. This enhancement would benefit in the construction of the active layer of photovoltaic solar cell to increase their efficiency. The second cause of SPR band broadening can be the fluctuations of dielectric permittivity of the surrounding medium $[46,47]$. Such fluctuations are originated from the random location of CdS NPs in the vicinity of $\mathrm{Au}$ ones. These fluctuations cause fluctuations of SPR frequencies in different gold NPs, which leads to the spectral broadening of SPR absorption band.

Room temperature PL studies were performed as well. The PL spectrum of the CdS NPs in D-g-PAA (Figure 6, blue dotted line) exhibits three bands labeled as $\mathrm{XL}_{\mathrm{s}}, \mathrm{XL}_{\mathrm{a}}$, and $\mathrm{XD}$ with maxima at $415 \mathrm{~nm}, 477 \mathrm{~nm}$, and $650 \mathrm{~nm}$, respectively. $\mathrm{XL}_{\mathrm{s}}$ and $\mathrm{XL}_{\mathrm{a}}$ bands correspond to the respective absorption $\mathrm{XA}_{\mathrm{s}}$ and $\mathrm{XA}_{\mathrm{a}}$ ones and are due to the radiative transitions in CdS NPs between the first excited and ground exciton states in single CdS NPs and aggregated ones. Also, an additional
$\mathrm{XD}$ band is due to radiative transitions in CdS NP excitons localized on the defects [48]. Taking into account that defect $\mathrm{XD}$ band dominates in the spectrum, one can conclude that the synthesized CdS NPs have quite defected structure. Since CdS NPs are quite small, it is reasonable to assume that these defects are surface ones. The PL spectrum of $\mathrm{Au} / \mathrm{CdS} / \mathrm{D}$ g-PAA hybrids dispersed in water is shown in Figure 6 by red solid line. This spectrum is quite surprising; namely, the intensity of localized excitons' XD band decreased hugely (6.5 times), while the total intensity of free excitons' $\mathrm{XL}_{\mathrm{s}}$ and $\mathrm{XL}_{\mathrm{a}}$ bands increased significantly (3.2 times). It is interesting that a similar result was obtained when the solution of Au NPs in D-g-PAA was mixed with the solution of CdS NPs in D-gPAA (Au+CdS/D-g-PAA mixture). The PL spectrum of such mixture is shown in Figure 6 by green dash-dotted line. One can see that the intensity of XD band decreases (12 times) and the total intensity of $\mathrm{XL}_{\mathrm{s}}$ and $\mathrm{XL}_{\mathrm{a}}$ ones increases (4 times) even more than for $\mathrm{Au} / \mathrm{CdS} / \mathrm{D}$-g-PAA hybrids. One can suppose that so strong decrease of defect XD band is due to some passivation of the surface of small CdS NPs carried out by Au ones. Indeed, as we noted above in $\mathrm{Au} / \mathrm{CdS} / \mathrm{D}$-g-PAA system, 


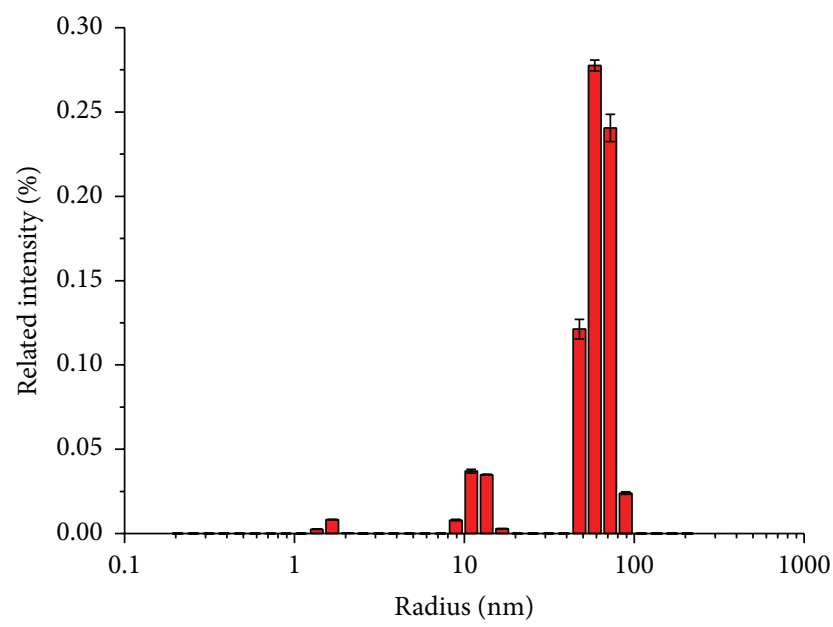

(a)

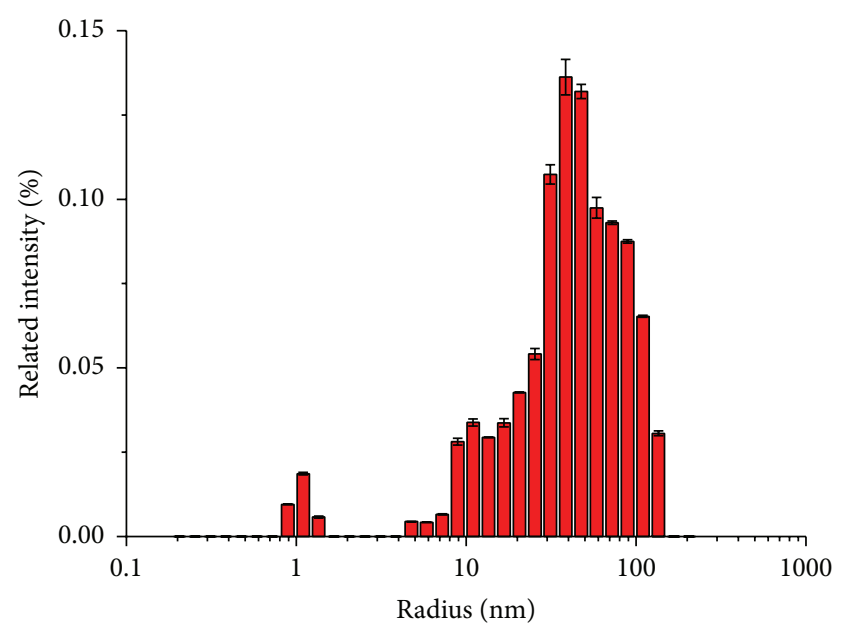

(b)

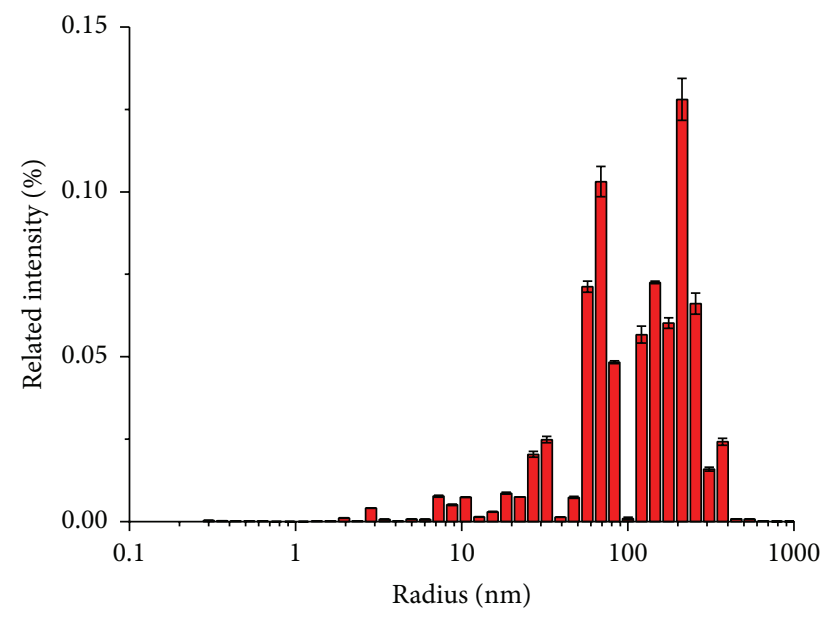

(c)

Figure 4: NPs' hydrodynamic radii distribution in $\mathrm{Au}(\mathrm{a}), \mathrm{CdS}(\mathrm{b})$, and Au/CdS (c) sols synthesized in D-g-PAA matrix.

the small CdS NPs are located around the larger Au ones. In $\mathrm{Au} / \mathrm{CdS} / \mathrm{D}$-g-PAA composite the CdS NPs are located in the vicinity of Au ones (the distances between Au NPs and CdS ones are several tens of $\mathrm{nm}$, which is seen in TEM image, Figure 3). Thus, CdS NPs are located in the dipolar plasmonic field of $\mathrm{Au}$ NPs which enhances the exciton radiative transitions in CdS ones. Such enhancement leads to significant increase of intensity of PL of free excitons in CdS NPs.

The increase of intensity of the free exciton $\mathrm{XL}_{\mathrm{s}}$ and $\mathrm{XL}_{\mathrm{a}}$ bands for $\mathrm{Au}+\mathrm{CdS} / \mathrm{D}$-g-PAA mixture and the decrease of defect XD band show that CdS NPs are located in the vicinity of Au ones. Note that it looks quite surprisingly since both CdS and Au NPs are surrounded by polymers which would cause the steric repulsion for NPs. To check the fact that $\mathrm{CdS}$ and $\mathrm{Au}$ NPs are located close to each other we measured the absorption spectrum of the $\mathrm{CdS}+\mathrm{Au} / \mathrm{D}-\mathrm{g}-\mathrm{PAA}$ mixture (green dash-dotted line in Figure 5). One can see that SPR band is red shifted and broadened in CdS+Au/D-g-PAA system comparing to SPR one in Au/D-g-PAA system (orange dashed line) which is similar to the behaviour of SPR band in hybrid CdS/Au/D-g-PAA system (red solid line). As it is noted above, such shift and broadening reflect the influence of CdS NPs on the SPR in Au ones proving the fact that CdS $\mathrm{NPs}$ are located in the vicinity of $\mathrm{Au}$ ones. Thus, one can conclude that in Au+CdS/D-g-PAA mixture the diffusion of $\mathrm{CdS}$ and $\mathrm{Au}$ NPs occurs leading to close location of these NPs in resulting mixture nanocomposite.

\section{Conclusions}

In conclusion, the metal/semiconductor (Au/CdS/D-g-PAA) nanocomposites were synthesized in the solution of branched D-g-PAA polymer. Polymer controlled the size and morphology of NPs in the process of their synthesis and was a stabilizing agent for sols. The nanosystems containing only Au NPs or only $\mathrm{CdS}$ ones were obtained for comparative studies. TEM and DLS of $\mathrm{Au} / \mathrm{CdS} / \mathrm{D}$-g-PAA nanocomposites results were in good agreement and revealed complicated nanocomposite structure consisting of isolated and aggregated Au NPs surrounded by small CdS NPs. These nanocomposites formed the aggregates-clusters with average size of 50-800 $\mathrm{nm}$. It can be also concluded that $\mathrm{Au} / \mathrm{CdS} / \mathrm{D}-\mathrm{g}-\mathrm{PAA}$ nanocomposites 


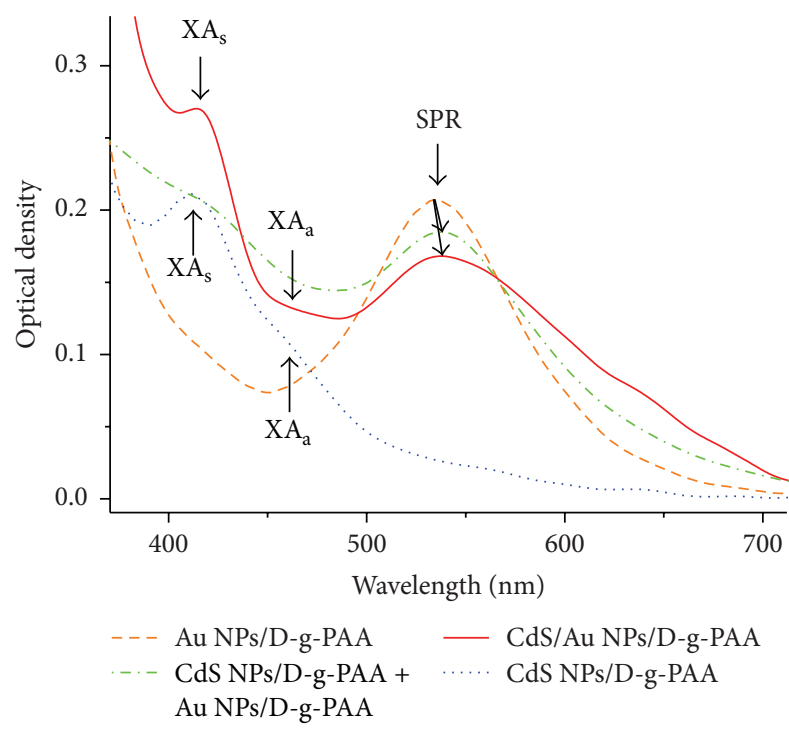

Figure 5: Absorption spectra of Au/CdS/D-g-PAA hybrids (red solid line), Au/D-g-PAA (orange dashed line), CdS/D-g-PAA (blue dotted line), and mixture of CdS/D-g-PAA and Au/D-g-PAA (green dash-dotted line) dispersed in water.

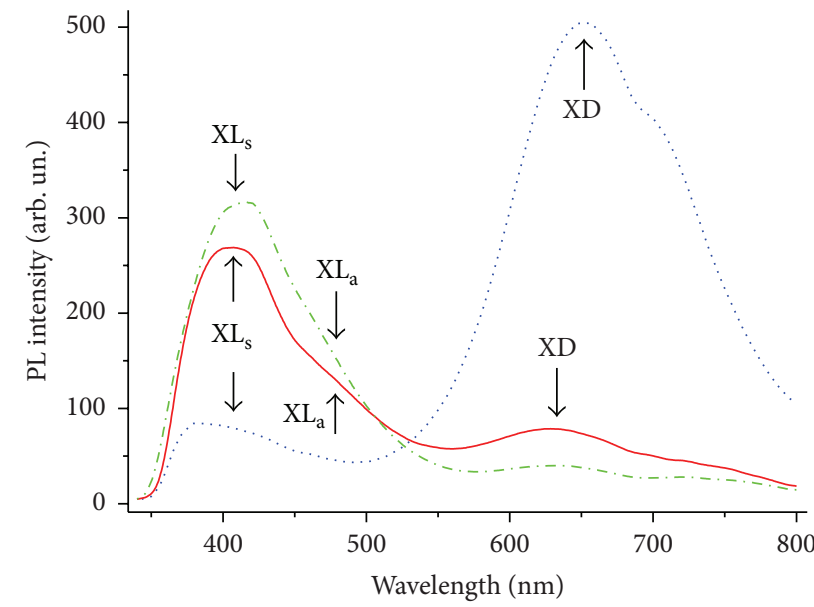

$\begin{array}{lll}\text { …. CdS NPs/D-g-PAA } & \text { CdS NPs/D-g-PAA + } \\ - \text { CdS/Au NPs/D-g-PAA } & \text { Au NPs/D-g-PAA }\end{array}$

Figure 6: PL spectra of CdS/D-g-PAA (blue dotted line), $\mathrm{Au} / \mathrm{CdS} / \mathrm{NPs} / \mathrm{D}$-g-PAA hybrids (red solid line), and mixture of CdS/D-g-PAA and Au/D-g-PAA NPs (green dash-dotted line) dispersed in water.

form the clusters localized in limited macromolecule volume. Optical absorption spectrum of Au/CdS/D-g-PAA nanocomposites exhibits three absorption bands at $415 \mathrm{~nm}, 460 \mathrm{~nm}$, and $548 \mathrm{~nm}$ that originate from the excitation of excitons in single and aggregated CdS NPs and surface plasmons in Au ones, respectively. The surface plasmon band of gold NPs red shifts and broadens in $\mathrm{Au} / \mathrm{CdS} / \mathrm{D}$-g-PAA nanocomposites comparing to the case of sole Au NPs in Au/D-g-PAA. This is due to the fact that in $\mathrm{Au} / \mathrm{CdS} / \mathrm{D}$-g-PAA system the gold NPs are surrounded by small CdS ones, which leads to increase of the local dielectric permittivity of environment of Au NPs.
The PL spectra of Au/CdS/D-g-PAA nanocomposites consist of three bands at $415 \mathrm{~nm}, 477 \mathrm{~nm}$, and $650 \mathrm{~nm}$, respectively, that correspond to radiative transitions in free excitons excited in single and aggregated CdS NPs and the excitons localized on lattice defects in CdS ones. A quite unexpected result was obtained, which is that in the presence of Au NPs the intensity of PL band of localized excitons in CdS NPs decreases hugely (6.5 times) while the intensity of PL bands of the free excitons sufficiently increases (3.2 times). Most probably, the strong decrease of localized exciton band is due to passivation of the surface of small CdS NPs carried out by the $\mathrm{Au}$ ones. Meanwhile, the significant increase of intensity of PL of free excitons is due to the enhancement of radiative excitonic transitions in CdS NPs by field of surface plasmon in $\mathrm{Au}$ ones.

\section{Competing Interests}

The authors declare that they have no competing interests.

\section{Acknowledgments}

O. A. Yeshchenko and I. S. Bondarchuk acknowledge the support by the NATO Science for Peace and Security (SPS) Program (Grant NUKR.SFPP 984617).

\section{References}

[1] A. P. Alivisatos, "Semiconductor clusters, nanocrystals, and quantum dots," Science, vol. 271, no. 5251, pp. 933-937, 1996.

[2] X. G. Peng, L. Manna, W. D. Yang et al., "Shape control of CdSe nanocrystals," Nature, vol. 404, no. 6773, pp. 59-61, 2000.

[3] Z. A. Peng and X. Peng, "Formation of high-quality CdTe, CdSe, and CdS nanocrystals using $\mathrm{CdO}$ as precursor," Journal of the American Chemical Society, vol. 123, no. 1, pp. 183-184, 2001.

[4] Y. Li, E. C. Y. Liu, N. Pickett et al., "Synthesis and characterization of CdS quantum dots in polystyrene microbeads," Journal of Materials Chemistry, vol. 15, no. 12, pp. 1238-1243, 2005.

[5] E. D. Sone and S. I. Stupp, "Semiconductor-encapsulated peptide-amphiphile nanofibers," Journal of the American Chemical Society, vol. 126, no. 40, pp. 12756-12757, 2004.

[6] H. Liang, T. E. Angelini, P. V. Braun, and G. C. L. Wong, "Roles of anionic and cationic template components in biomineralization of CdS nanorods using self-assembled DNA-membrane complexes," Journal of the American Chemical Society, vol. 126, no. 43, pp. 14157-14165, 2004.

[7] N. L. Rosi and C. A. Mirkin, "Nanostructures in biodiagnostics," Chemical Reviews, vol. 105, no. 4, pp. 1547-1562, 2005.

[8] E. Ozbay, "Plasmonics: merging photonics and electronics at nanoscale dimensions," Science, vol. 311, no. 5758, pp. 189-193, 2006.

[9] W. L. Barnes, A. Dereux, and T. W. Ebbesen, "Surface plasmon subwavelength optics," Nature, vol. 424, pp. 824-830, 2003.

[10] M. I. Stockman, "Nanoplasmonics: past, present, and glimpse into future," Optics Express, vol. 19, no. 22, pp. 22029-22106, 2011.

[11] Y. Fang, N.-H. Seong, and D. D. Dlott, "Measurement of the distribution of site enhancements in surface-enhanced raman scattering," Science, vol. 321, no. 5887, pp. 388-392, 2008.

[12] F. Le, D. W. Brandl, Y. A. Urzhumov et al., "Metallic nanoparticle arrays: a common substrate for both surface-enhanced Raman 
scattering and surface-enhanced infrared absorption," ACS Nano, vol. 2, no. 4, pp. 707-718, 2008.

[13] G. Schatz, M. Young, R. Van, and R. Van Duyne, "Electromagnetic mechanism of SERS," in Surface-Enhanced Raman Scattering: Physics and Applications, K. Kneip, M. Moskovits, and H. Kneip, Eds., vol. 103 of Topics in Applied Physics, pp. 1945, Springer, New York, NY, USA, 2006.

[14] T. Ming, H. Chen, R. Jiang, Q. Li, and J. Wang, "Plasmoncontrolled fluorescence: beyond the intensity enhancement," The Journal of Physical Chemistry Letters, vol. 3, no. 2, pp. 191202, 2012.

[15] O. L. Muskens, V. Giannini, J. A. Sánchez-Gil, and J. Gómez Rivas, "Strong enhancement of the radiative decay rate of emitters by single plasmonic nanoantennas," Nano Letters, vol. 7, no. 9, pp. 2871-2875, 2007.

[16] P. Anger, P. Bharadwaj, and L. Novotny, "Enhancement and quenching of single-molecule fluorescence," Physical Review Letters, vol. 96, Article ID 113002, 2006.

[17] S. Kühn, U. Häkanson, L. Rogobete, and V. Sandoghdar, "Enhancement of single-molecule fluorescence using a gold nanoparticle as an optical nanoantenna," Physical Review Letters, vol. 97, no. 1, Article ID 017402, 2006.

[18] O. A. Yeshchenko, I. S. Bondarchuk, V. V. Kozachenko, and M. Y. Losytskyy, "Photoluminescence of rhodamine 6G in plasmonic field of Au nanoparticles: temperature effects," Journal of Luminescence, vol. 158, pp. 294-300, 2015.

[19] W.-T. Chen, T.-T. Yang, and Y.-J. Hsu, "Au-CdS core-shell nanocrystals with controllable shell thickness and photoinduced charge separation property," Chemistry of Materials, vol. 20, no. 23, pp. 7204-7206, 2008.

[20] W. Zhang, J. Zheng, C. Tan et al., "Designed self-assembled hybrid Au@CdS core-shell nanoparticles with negative charge and their application as highly selective biosensors," Journal of Materials Chemistry B, vol. 3, pp. 217-224, 2015.

[21] Y. Liu, X. Feng, J. Shen, J.-J. Zhu, and W. Hou, "Fabrication of a novel glucose biosensor based on a highly electroactive polystyrene/polyaniline/Au nanocomposite," The Journal of Physical Chemistry B, vol. 112, no. 30, pp. 9237-9242, 2008.

[22] J. Li, S. K. Cushing, P. Zheng et al., "Solar hydrogen generation by a CdS-Au- $\mathrm{TiO}_{2}$ sandwich nanorod array enhanced with au nanoparticle as electron relay and plasmonic photosensitizer," Journal of the American Chemical Society, vol. 136, no. 23, pp. 8438-8449, 2014.

[23] Q. Gao, J. Han, and Z. Ma, "Polyamidoamine dendrimerscapped carbon dots/Au nanocrystal nanocomposites and its application for electrochemical immunosensor," Biosensors and Bioelectronics, vol. 49, pp. 323-328, 2013.

[24] R. Costi, A. E. Saunders, E. Elmalem, A. Salant, and U. Banin, "Visible light-induced charge retention and photocatalysis with hybrid CdSe-Au nanodumbbells," Nano Letters, vol. 8, no. 2, pp. 637-641, 2008.

[25] G. Jie, P. Liu, L. Wang, and S. Zhang, "Electrochemiluminescence immunosensor based on nanocomposite film of $\mathrm{CdS}$ quantum dots-carbon nanotubes combined with gold nanoparticles-chitosan," Electrochemistry Communications, vol. 12, no. 1, pp. 22-26, 2010.

[26] H. Yu, M. Chen, P. M. Rice, S. X. Wang, R. L. White, and S. Sun, "Dumbbell-like bifunctional $\mathrm{Au}-\mathrm{Fe}_{3} \mathrm{O}_{4}$ nanoparticles," Nano Letters, vol. 5, no. 2, pp. 379-382, 2005.

[27] W. L. Shi, H. Zeng, Y. Sahoo et al., "A general approach to binary and ternary hybrid nanocrystals," Nano Letters, vol. 6, no. 4, pp. 875-881, 2006.
[28] D. Steiner, T. Mokari, U. Banin, and O. Millo, "Electronic structure of metal-semiconductor nanojunctions in gold CdSe nanodumbbells," Physical Review Letters, vol. 95, no. 5, Article ID 056805, 2005.

[29] J. Prasad Rao and K. E. Geckeler, "Polymer nanoparticles: preparation techniques and size-control parameters," Progress in Polymer Science, vol. 36, no. 7, pp. 887-913, 2011.

[30] M. Bezuglyi, N. Kutsevol, M. Rawiso, and T. Bezugla, "Watersoluble branched copolymers dextran-polyacrylamide and their anionic derivates as matrices for metal nanoparticles insitu synthesis," Chemik, vol. 66, no. 8, pp. 862-867, 2012.

[31] V. Chumachenko, N. Kutsevol, M. Rawiso, and C. Schmutz, "In situ formation of silver nanoparticles in linear and branched polyelectrolyte matrices using various reducing agents," Nanoscale Research Letters, vol. 9, article 164, 2014.

[32] N. V. Kutsevol, V. A. Chumachenko, M. Rawiso, V. F. Shkodich, and O. V. Stoyanov, "Star-like dextran-polyacrylamide polymers: Prospects of use in nanotechnologies," Journal of Structural Chemistry, vol. 56, no. 5, pp. 959-966, 2015.

[33] N. Kutsevol, M. Bezuglyi, T. Bezugla, and M. Rawiso, "Synthesis of silver nanoparticles in star-like Dextran-graftpolyacrylamide matrices," Molecular Crystals and Liquid Crystals, vol. 590, no. 1, pp. 179-185, 2014.

[34] N. Kutsevol, T. Bezugla, M. Bezuglyi, and M. Rawiso, "Branched dextran-graft-polyacrylamide copolymers as perspective materials for nanotechnology," Macromolecular Symposia, vol. 317318, no. 1, pp. 82-90, 2012.

[35] N. Kutsevol, J.-M. Guenet, N. Melnik, D. Sarazin, and C. Rochas, "Solution properties of dextran-polyacrylamide graft copolymers," Polymer, vol. 47, no. 6, pp. 2061-2068, 2006.

[36] N. V. Kutsevol, T. N. Bezuglaya, and M. Y. Bezuglyi, "Features of the intramolecular structure of branched polymer systems in solution," Journal of Structural Chemistry, vol. 55, no. 3, pp. 548559, 2014.

[37] S. W. Provencher, "CONTIN: a general purpose constrained regularization program for inverting noisy linear algebraic and integral equations," Computer Physics Communications, vol. 27, no. 3, pp. 229-242, 1982.

[38] A. Scotti, W. Liu, J. S. Hyatt et al., "The CONTIN algorithm and its application to determine the size distribution of microgel suspensions," The Journal of Chemical Physics, vol. 142, no. 23, Article ID 234905, 2015.

[39] H. S. Mansur, A. A. P. Mansur, and J. C. González, "Synthesis and characterization of CdS quantum dots with carboxylicfunctionalized poly (vinyl alcohol) for bioconjugation," Polymer, vol. 52, no. 4, pp. 1045-1054, 2011.

[40] H. S. Mansur, J. C. González, and A. A. P. Mansur, "Biomoleculequantum dot systems for bioconjugation applications," Colloids and Surfaces B: Biointerfaces, vol. 84, no. 2, pp. 360-368, 2011.

[41] X. Peng, J. Wickham, and A. P. Alivisatos, "Kinetics of II-VI and III-V colloidal semiconductor nanocrystal growth: "Focusing" of size distributions," Journal of the American Chemical Society, vol. 120, no. 21, pp. 5343-5344, 1998.

[42] S. Link, M. B. Mohamed, and M. A. El-Sayed, "Simulation of the optical absorption spectra of gold nanorods as a function of their aspect ratio and the effect of the medium dielectric constant," The Journal of Physical Chemistry B, vol. 103, no. 16, pp. 3073-3077, 1999.

[43] Y. Q. He, S. P. Liu, L. Kong, and Z. F. Liu, "A study on the sizes and concentrations of gold nanoparticles by spectra of absorption, resonance Rayleigh scattering and resonance nonlinear scattering," Spectrochimica Acta Part A: Molecular and 
Biomolecular Spectroscopy, vol. 61, no. 13-14, pp. 2861-2866, 2005.

[44] A. E. Saunders, I. Popov, and U. Banin, "Synthesis of hybrid CdS-Au colloidal nanostructures," Journal of Physical Chemistry B, vol. 110, no. 50, pp. 25421-25429, 2006.

[45] K. Easawi, M. Nabil, T. Abdallah, S. Negm, and H. Talaat, "Plasmonic absorption enhancement in $\mathrm{Au} / \mathrm{CdS}$ nanocomposite," World Academy of Science, Engineering and Technology, vol. 6, p. 25, 2012.

[46] U. Kreibig and M. Vollmer, Optical Properties of Metal Clusters, Springer, Berlin, Germany, 1995.

[47] S. A. Maier, Plasmonics: Fundamentals and Applications, Springer, New York, NY, USA, 2007.

[48] C. Barglik-Chory, C. Remenyi, C. Dem et al., "Synthesis and characterization of manganese-doped CdS nanoparticles," Physical Chemistry Chemical Physics, vol. 5, no. 8, pp. 1639-1643, 2003. 

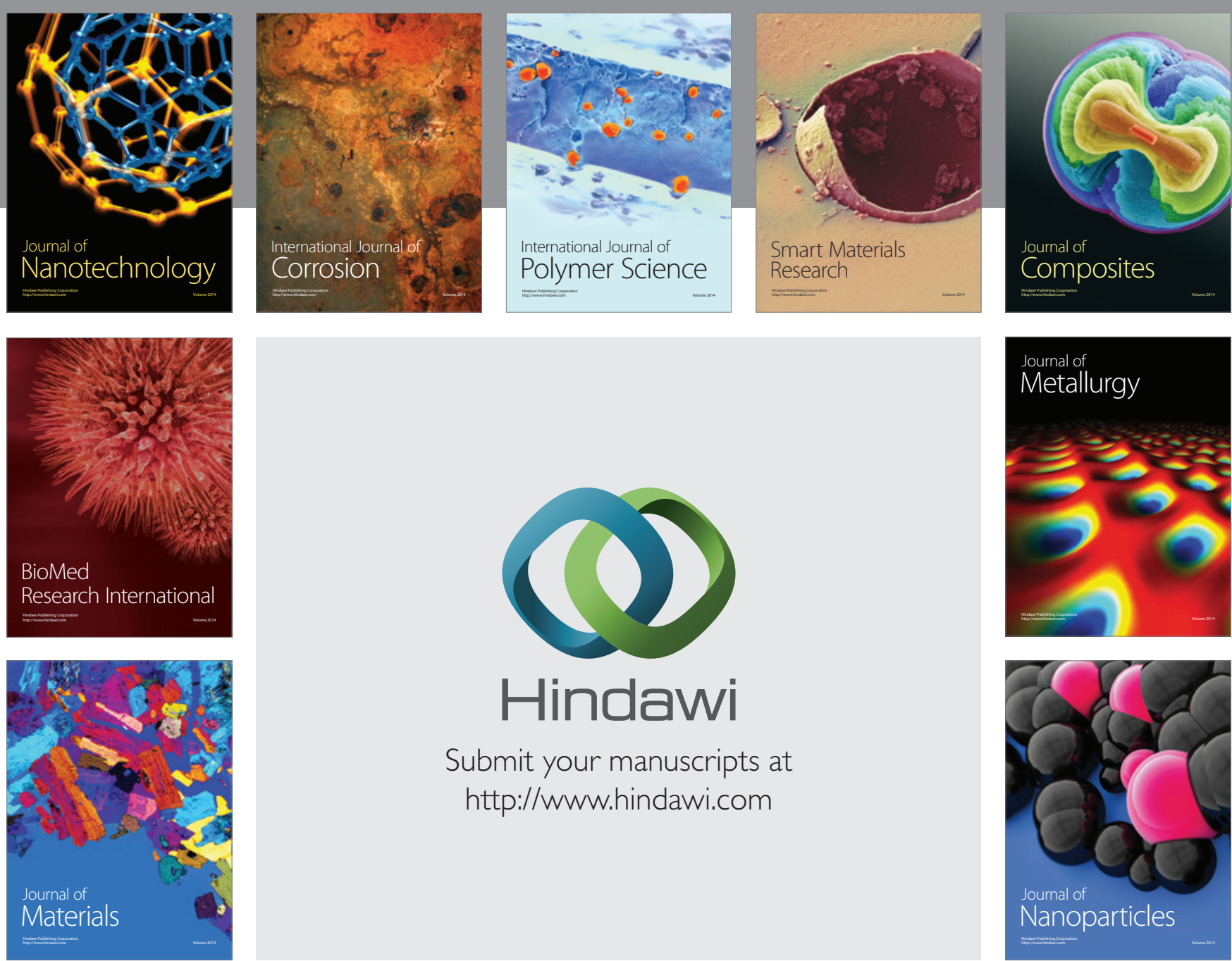

\section{Hindawi}

Submit your manuscripts at

http://www.hindawi.com

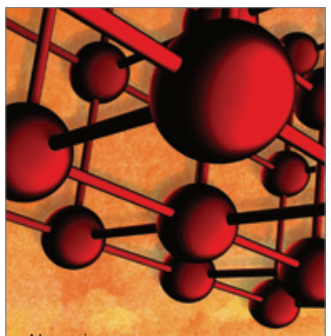

Materials Science and Engineering
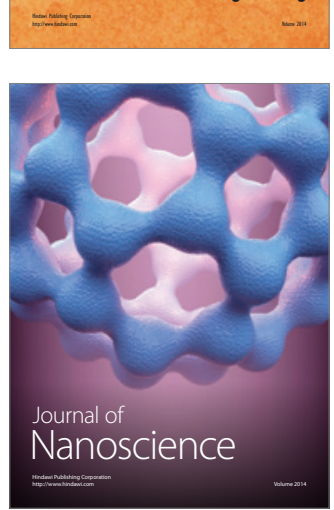
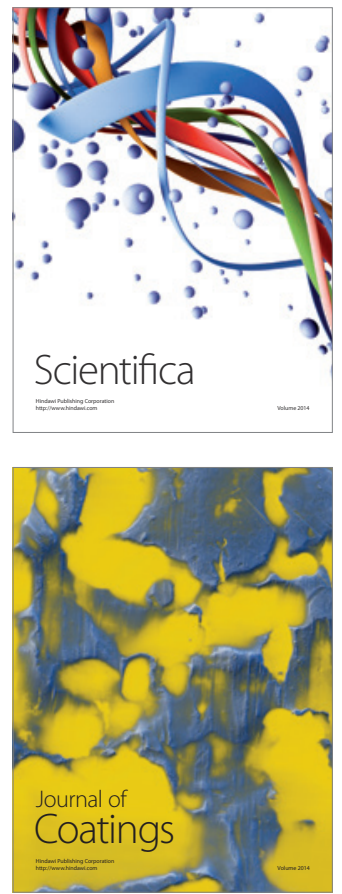
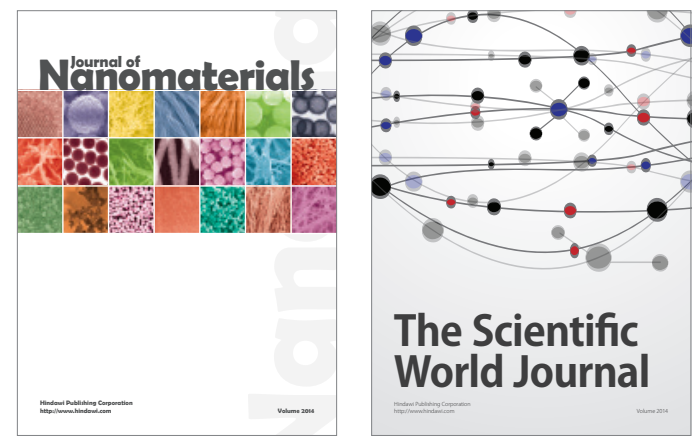

The Scientific World Journal
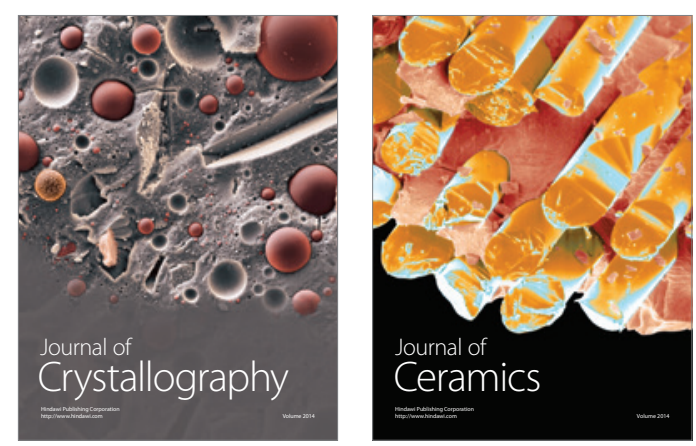
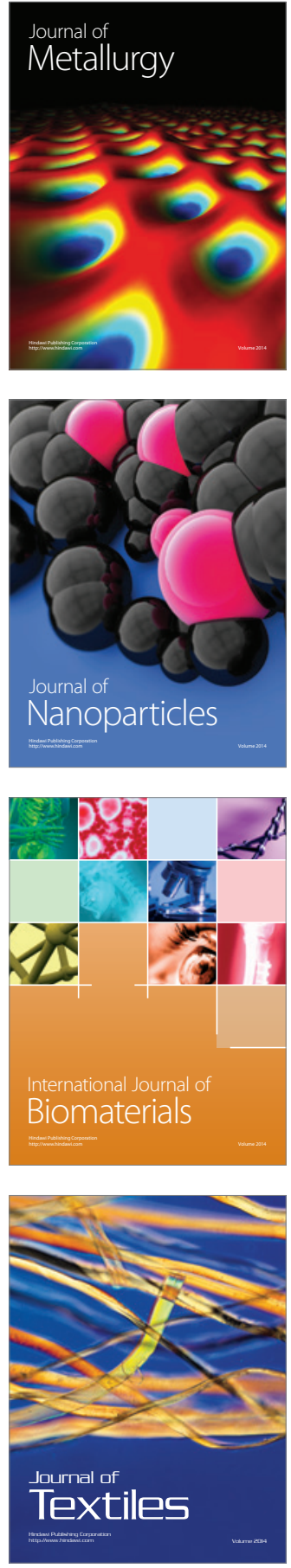